\title{
MATLAB ALGORITHM FOR DRIVING PATTERN DETECTION AND ANALYSIS USING SMARTPHONE SENSORS
}

\author{
${ }^{1}$ C.Edwin Samuel, ${ }^{1}$ Kapilesh Kathiresh, ${ }^{2}$ Balaji Ramachandran \\ ${ }^{1}$ Department of Automobile Engineering, Hindustan Institute of Technology and Science \\ OMR, Padur, Kelambakam, Chennai, Tamil Nadu 603103
}

${ }^{2}$ Department of Mechanical Engineering, Hindustan Institute of Technology \& Science, OMR, Padur, Kelambakam, Chennai, Tamil Nadu 603103

Corresponding Author: edwin14696@gmail.com, sridharabalaa@gmail.com

\begin{abstract}
:
In countries like India and China where the population density is very high and the number of vehicles on road keep on increasing every year it presents us with a problem that is monitoring this entire situation and preventing rash driving and accidents and even if they take place there should be immediate action taken to prevent it from happening again and making the roads a safer environment for everyone. To solve this problem, utilized sensors present in the Smartphone specifically the accelerometer sensor to detect the changes in parameters, for this an app was created. The app will read the sensor data that are embedded in the Smartphone and stores the sensors data in the database, The main reason of using the Smartphone is that implementation will be very easy and cost effective as the hardware is already available with everyone and the cost of implementation will be lower when compared to producing an exclusive hardware for this purpose only. The theoretical threshold values for accelerometer sensor data to calculate sudden breaking, sudden acceleration, harsh cornering with the respective axis is used to determine the initial situation. Feeding this data in MATLAB was we use an algorithm developed for this very purpose of analyzing this data.
\end{abstract}

Keywords: Sensors, Algorithm, Accelerometer, Driving pattern, Matlab

1.

\section{INTRODUCTION}

In the past 5 years smartphones have evolved into a small slab of technology that can be carried with us every day and almost everywhere and yet they possess so many sensors and processing power that now easily rivals the space craft that carried the first humans ever to set foot on the moon [1]. Driving a vehicle is something most of humans do in their lifetime but not everyone is good at it or following the rules in some situations and this compromises road safety [2]. Using something like a smartphone which everyone carries with them to detect their driving style to determine whether the driver is driving rashly and creating a road safety hazard, feeding this information through the algorithm specifically designed to analyse the data obtained from the driver's smartphone's accelerometer sensor processing it to get accurate data and using this data to warn both the driver and at a certain point the authorities to maintain a safe environment for both the drivers and pedestrians [3]. In this research the SUV vehicle user friendly analysis system had been implemented in Matlab/Simulink for investigating vehicle on dynamic process. Also the response of the body was analyzed at $0-10$ kmph, 0-20 kmph, 0-30 kmph, 0-40 kmph respectively under various conditions such as acceleration, braking and turning of vehicle have been analysed using MATLAB and the data for these events were put through the algorithm to obtain output graphs for these conditions and were analysed for the driving pattern. Frequency response was also analyzed using FFT (Fast Fourier Transform) Algorithm 
[3]. In this research, A model with 6-DOF had been created and analyzed to evaluate and optimize ride comfort and pitch angle. In the end a DOE method had been implemented to obtain maximum ride comfort from the best values of the varied parameters [4]. In Matlab, three mathematical models were developed to analyze and evaluate the effects of tire pressure on consumption of fuel, handling of the vehicle and ride characteristics [5]. Also evaluation in time and frequency domain was done for a 4DOF half car model that had been developed and subjected to sine input, step input and random road profile input Smartphones [6].

\section{$2 . \quad$ EXPERIMENTAL METHODOLOGY-DETERMINING THE DRIVING PATTERN}

\subsection{The Smartphone Sensor}

The smartphone sensor plays the primary role of data collection through the accelerometer sensor. The accelerometer sensor measures the change in acceleration in each axis or the $\mathrm{G}$ force. The accelerometer sensor used in phones are 6 DOF accelerometer sensor [1,7]. The driver's driving patter is captured through this sensor via an app that is specifically developed for this purpose and stored in a database which can be easily accessed [8].

The data collected from the accelerometer sensor is not accurate enough to decide whether the driver is driving rashly or has crashed because this captured data contains lot of noise that is created by many factors such as the smartphone not being fixed in a place rigidly or the movement of the part it was placed on. To solve this, data was created algorithm for proper and accurate analysis.

\subsection{Relation between the Vehicle Coordinate and Phone Coordinate System}

The $\mathrm{X}, \mathrm{Y}, \mathrm{Z}$ axis of the vehicle and the phone should be matched and the coordinate system differs for the vehicle and phone [2,9] as the vehicle coordinate system is used to measure factors like roll, pitch, yaw but here we match the vehicle coordinate system and the phone,s coordinate system and it represents:

1) $X$ axis: Lateral acceleration or the cornering of the vehicle

2) $Y$ axis: Longitudinal acceleration or the acceleration and braking of the vehicle

3) $\mathrm{Z}$ axis: Vertical acceleration or the bumps the vehicle encounters.
Using these axis we define whether the driver is accelerating, Braking, Cornering too harshly and the bumps and portholes on the road too. To determine the limit above which the driver is driving dangerously upon the need something is called the threshold value.

\section{THRESHOLD VALUE}

\subsection{Meaning of Threshold Value}

Every vehicle is designed according to the purpose its going to serve ie. There are different types of vehicles such as light motor vehicle, heavy motor vehicles, transportation vehicles etc. The main focus right now is on light motor. The design of the vehicle is different that also means that the external factors acting on it will also vary i.e. The loads acting on it will vary according to the type of vehicle. The main forces we are concerned with while trying to detect the driving pattern of the driver are the G-forces acting on the vehicle while accelerating, braking and cornering. The threshold value is the value that indicates that above that its considered rash driving or a crash.

\subsection{Calculating the Threshold Values}

To calculate the threshold values we have 2 methods and the use of both methods is done to increase the accuracy. The first one is theoretical calculation of threshold values which includes formulas, taking readings and iteration method. The second step in calculate the actual data from the graphs using algorithm and verify with it after all processes are complete. This is done to increase the accuracy.

\subsubsection{Calculating the Threshold Values by Theoretical Method}

To calculate the threshold values using the iteration method, tests are performed such as 0-10 Kmph, 0-20 Kmph and so on to get the time taken and the distance covered in that time. Same with braking. Now using the given formulas longitudinal acceleration (1) and lateral acceleration (2) is found out for different speed and the threshold value is determined from it. 


\subsubsection{Calculating the Threshold Values by Practical Method}

This comes after the theoretical method and in this practical method the data is put through the developed algorithm and the results from the graphs are then compared with the theoretical values and final threshold values are fixed. Now having the threshold values we can determine the driving pattern in the algorithm and clearly find out if the driver is driving rashly or has crashed, The threshold value can also be used in the app for initial warning to the driver [10].

\section{APPLICATION}

The main purpose of the application is to collect the accelerometer sensor data. It starts recording the data when the application is opened and started. The phone must be placed on a flat and rigid surface and it should be fixed in that place so that it doesn't move. The application also gives the driver initial warning if the driver is exceeding the threshold limit and after the data is properly processed in the algorithm and the application can also notify the authorities if needed.

\section{ALGORITHM}

The algorithm was specifically developed to processes the raw data and analyse it to determine the driving pattern of the driver. Raw data from the phone's accelerometer sensor contains lot of high frequency noise doesn't give an accurate data so it needs to be put through many processes in the matlab algorithm. The algorithm is compatible not just with smartphone's accelerometer sensor but it can also work with other accelerometer sensors such as the ones used with arduino and raspberry pi but it has to be a $6 \mathrm{DOF}$ accelerometer sensor [11].

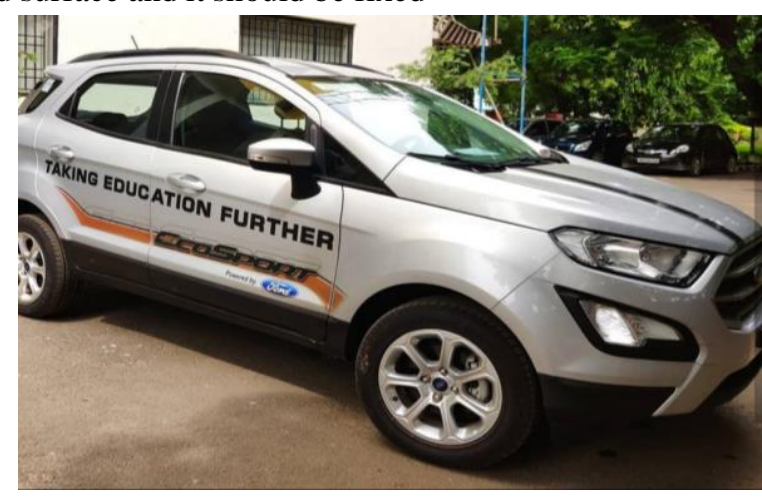

Fig.1 Vehicle for Test Drives

\subsection{Filtering the Raw Data}

The raw data contains lot of high frequency noise as seen in figure 1 which doesn't give use accurate data to work with so the first step in the algorithm is to filter the data. The red signal represents raw data, blue signal filtered data and green line the threshold value.

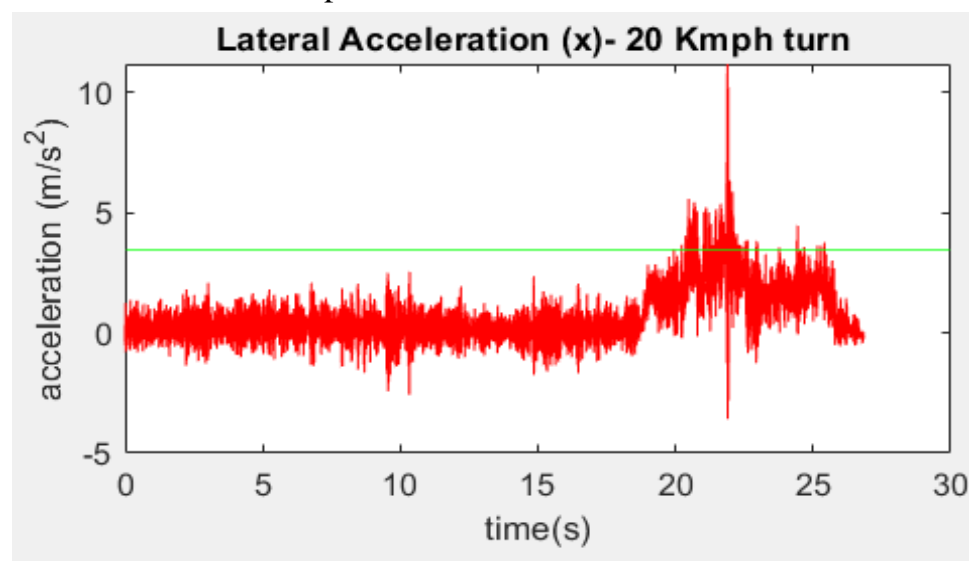

Fig.2 Graph of Raw Data

The filter used in the algorithm is a low pass filter high filters out all the high frequencies and gives an accurate data to work with. First the raw data is converted to frequency domain from time domain by applying Fast Fourier Transformation (FFT) to find the cut off frequency which is used in the filter to filter out high frequency noise. In figure 2 the difference 
between the raw and filtered data can be seen compared to figure 2, here the green line represents the threshold value. One more important factor when it comes to filtering is to prevent phase shift after filtering the raw data and the algorithm has provision to prevent this phase shift [12].

\subsection{Plotting above Threshold Limit}

After filtering the raw data the algorithm proceeds to the next step that is to find points where the driver has exceeded the threshold limit and to mark them on the graph indicating the exact values in $\mathrm{m} / \mathrm{s}^{2}$ and the exact time when it has happened. The algorithm does everything automatically, The only thing is to feed the data in the algorithm and it does everything from filtering to finding points above threshold limit for acceleration, braking and cornering and plots the graphs for easy understanding as seen in figure 3 and 4 the values exceeding the threshold limit are marked on the graph.

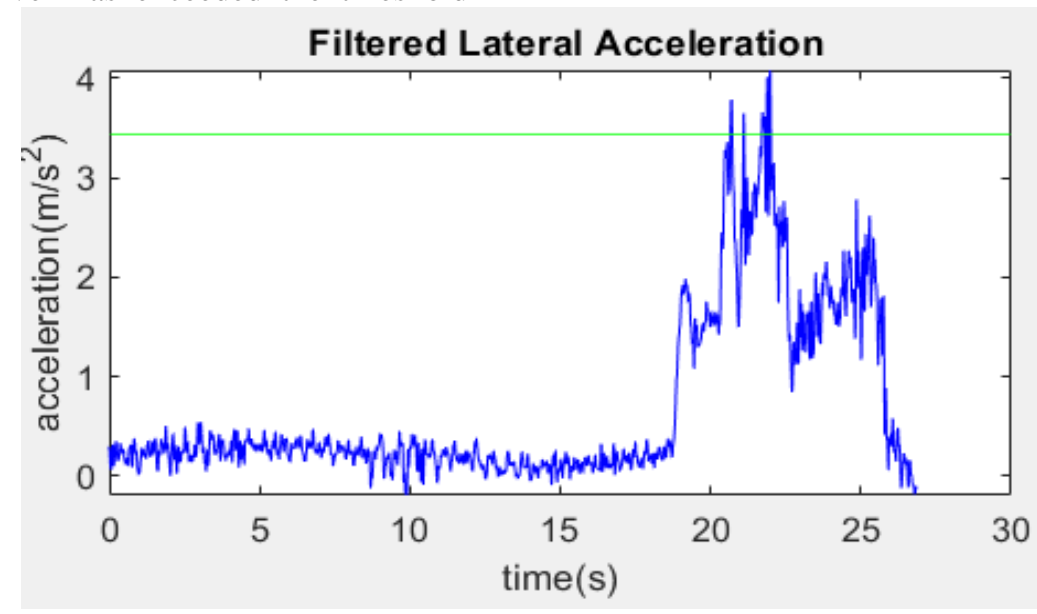

Fig.3 Filtered Data

This takes place for the entire 3 axis and simultaneously and the graphs are obtained together at the same time. The analysis of data can be done by anyone by looking at these graphs and using them it can be determined if the driver was driving rash $[12,13]$ or not or the vehicle has met with an accident, This data is then sent to the application gives the driver warnings for rash driving and if a crash has taken place notifies the authorities immediately

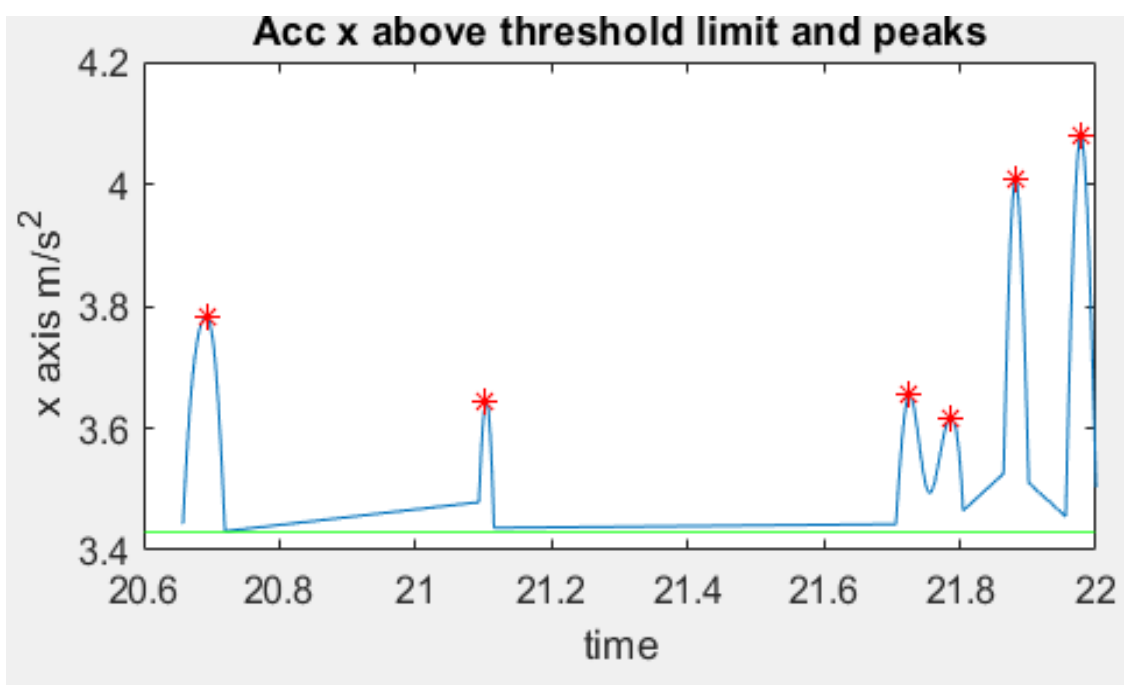

Fig.4 Points Above Threshold Limit

\subsection{Flow Chart for the Algorithm}

The step by step process for the algorithm is given in figure 4 where the algorithm starts off by taking in the raw data obtained from the phone's accelerometer sensor in which is in excel format and bringing it in the algorithm in matrix format. Then the raw data gets filtered using low pass filter and the phase shift is prevented. After filtering all 3 axis the 
threshold line is which is different for the 3 different axis is showed in the graph and the next step is plotting everything above the threshold limit to indicate points where the threshold limit was exceeded and my how much

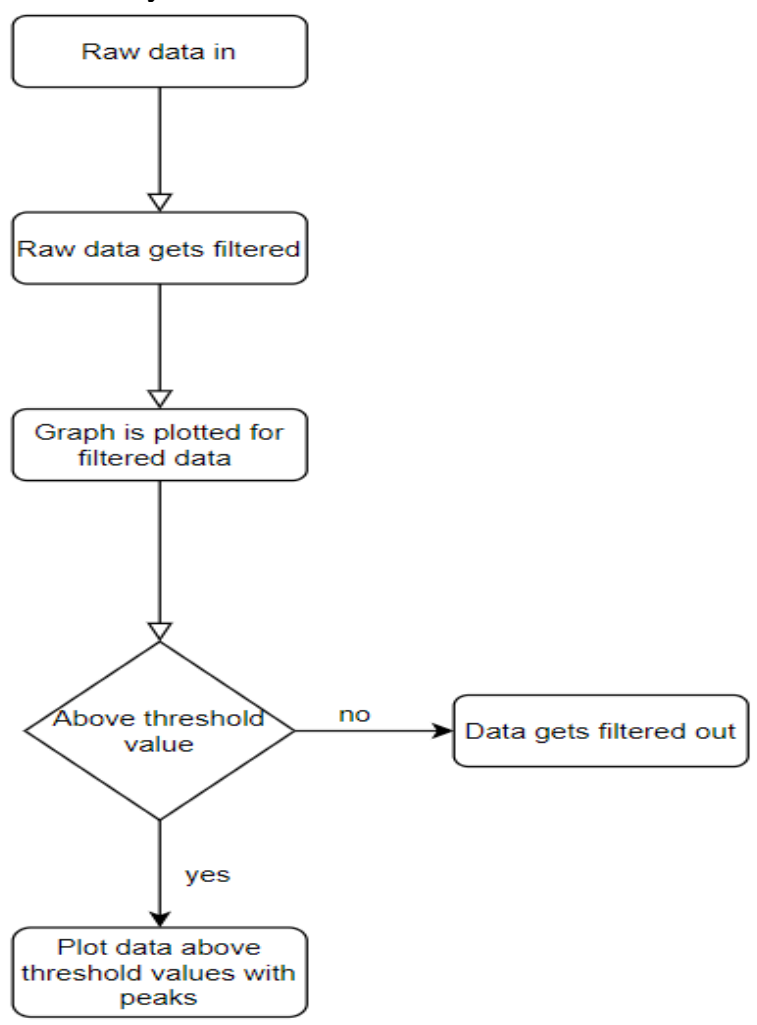

Fig. 4.1 Flow Chart for the Algorithm

\subsection{Output Graphs for Various Conditions}

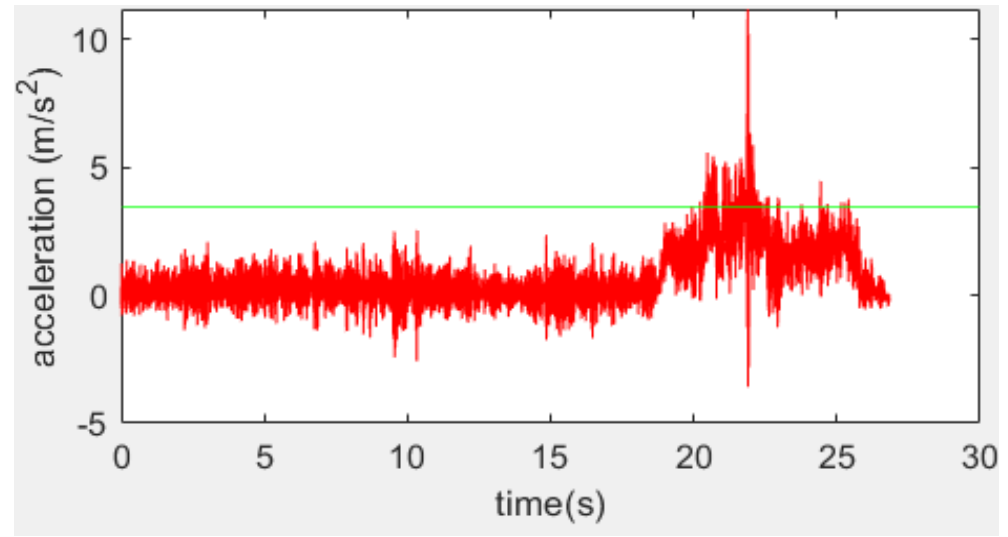

Fig.5 First Step Raw Data

Figure 5 depicts the raw signal from the phone's accelerometer where the red signal represents the raw unfiltered signal and the green line represents the threshold value. 


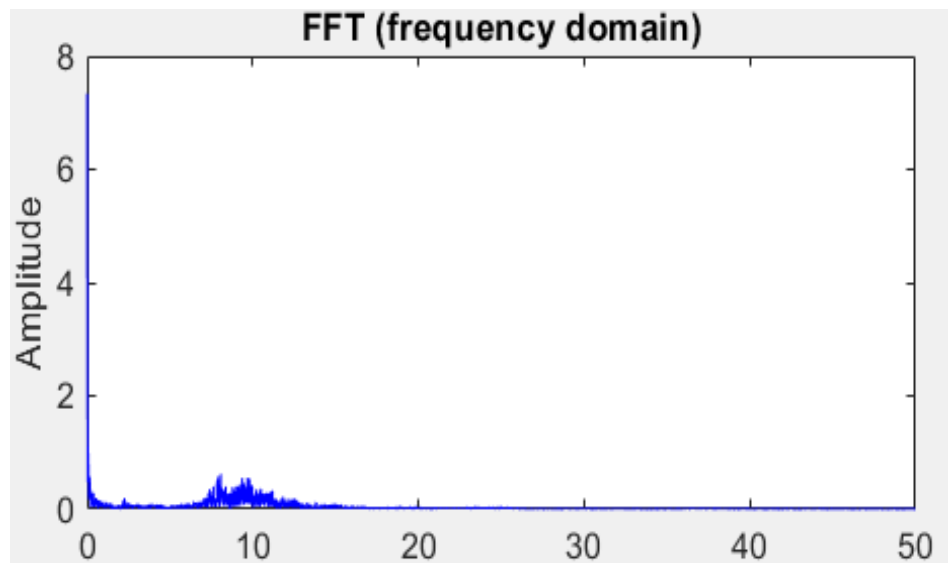

Fig.6 Second step FFT

Figure 6 depicts the second step in the filtering process where the raw data is converted from time domain to frequency domain to find out the high frequency noise and after which the low pass filter is applied on it to eliminate high frequency signal is the noise in the signal.

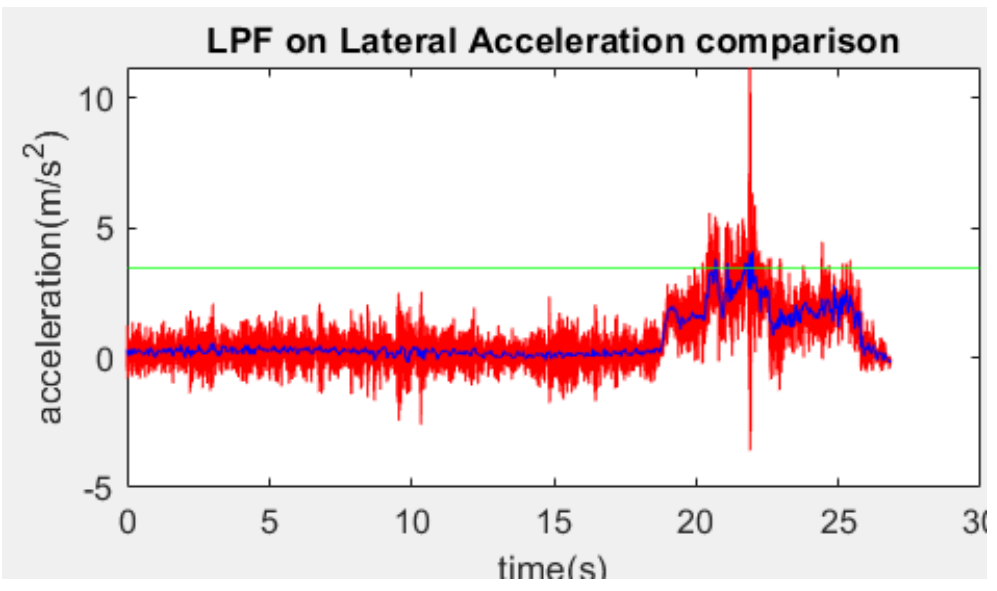

Fig.7 Third step Raw Data vs. Filtered Data

Figure 7 depicts the comparison between the unfiltered signal which is represented by red colour and filtered signal is represented by blue colour.

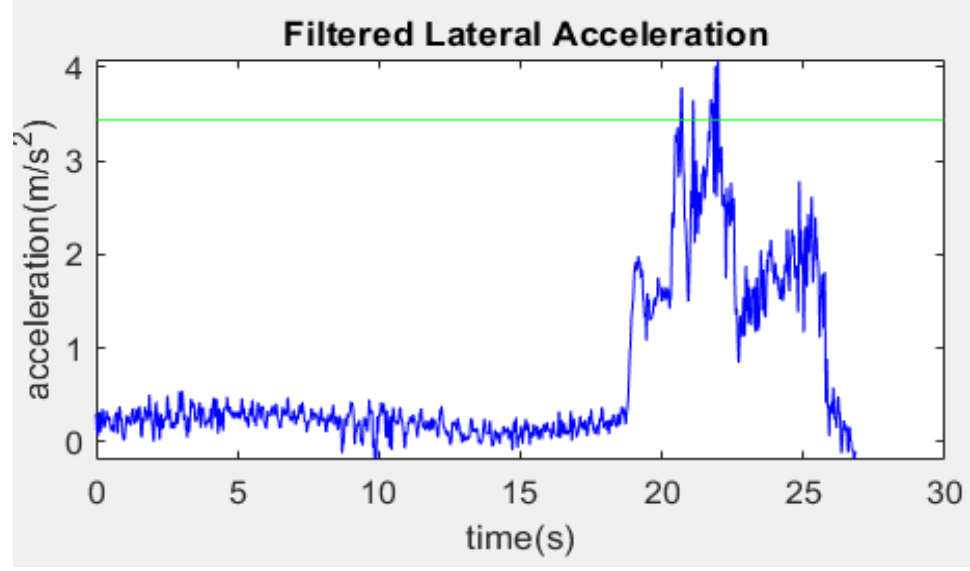

Fig.8 Final Step Filtered

Figure 8 depicts the final filtered signal using this filtering process which can now be used to find out points above the threshold limits. 

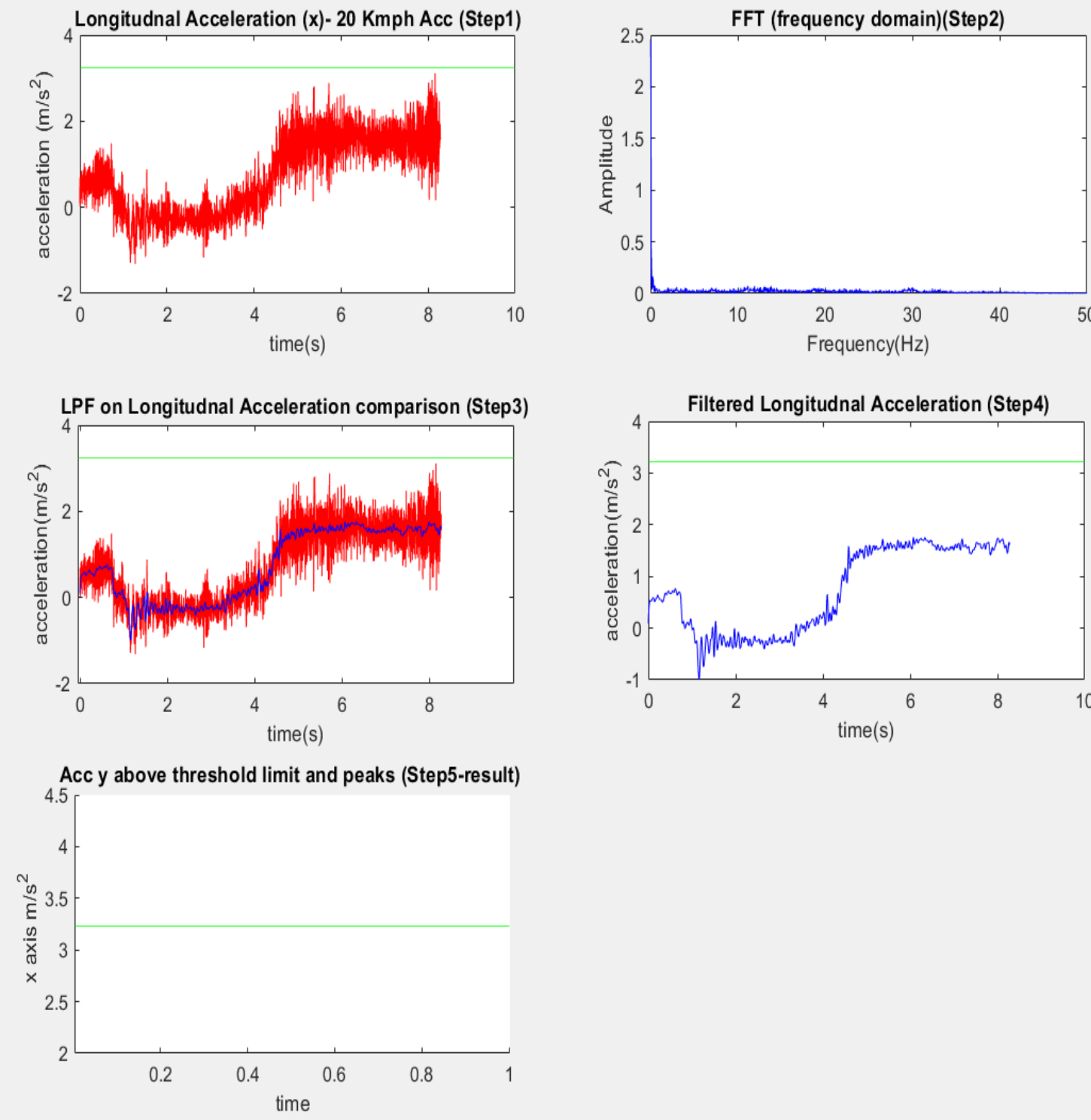

Fig.9 At $20 \mathrm{Kmph}$ acceleration complete output graph

Figure 9 depicts a scenario where the vehicle accelerates from 0-20 Kmph and the signal acquired from this event is processed by the algorithm which is shown graphically step by step. The red signal is unfiltered raw data and the blue signal is the filtered signal. The green line represents the threshold value $(0.33 \mathrm{~g})$ for acceleration above that is considered to be rash driving. 

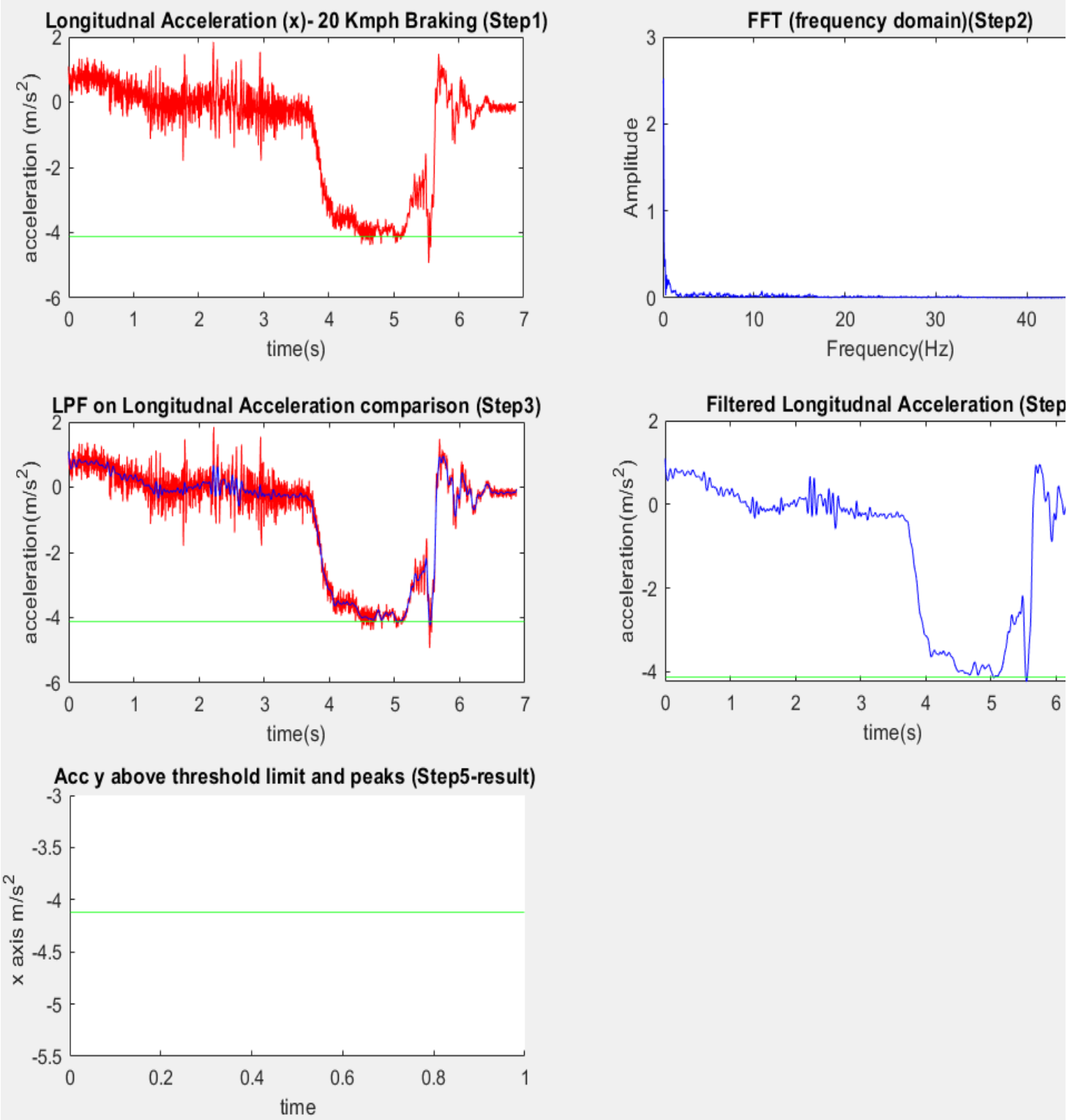

Fig.10 At $20 \mathrm{Kmph}$ braking complete output graph

Figure 10 depicts a scenario the vehicle brakes from 20-0 Kmph and the signal acquired from this event is processed by the algorithm which is shown graphically step by step. The red signal is unfiltered raw data and the blue signal is the filtered signal. The green line represents the threshold value $(0.42 \mathrm{~g})$ for acceleration above which is considered to be rash driving. 

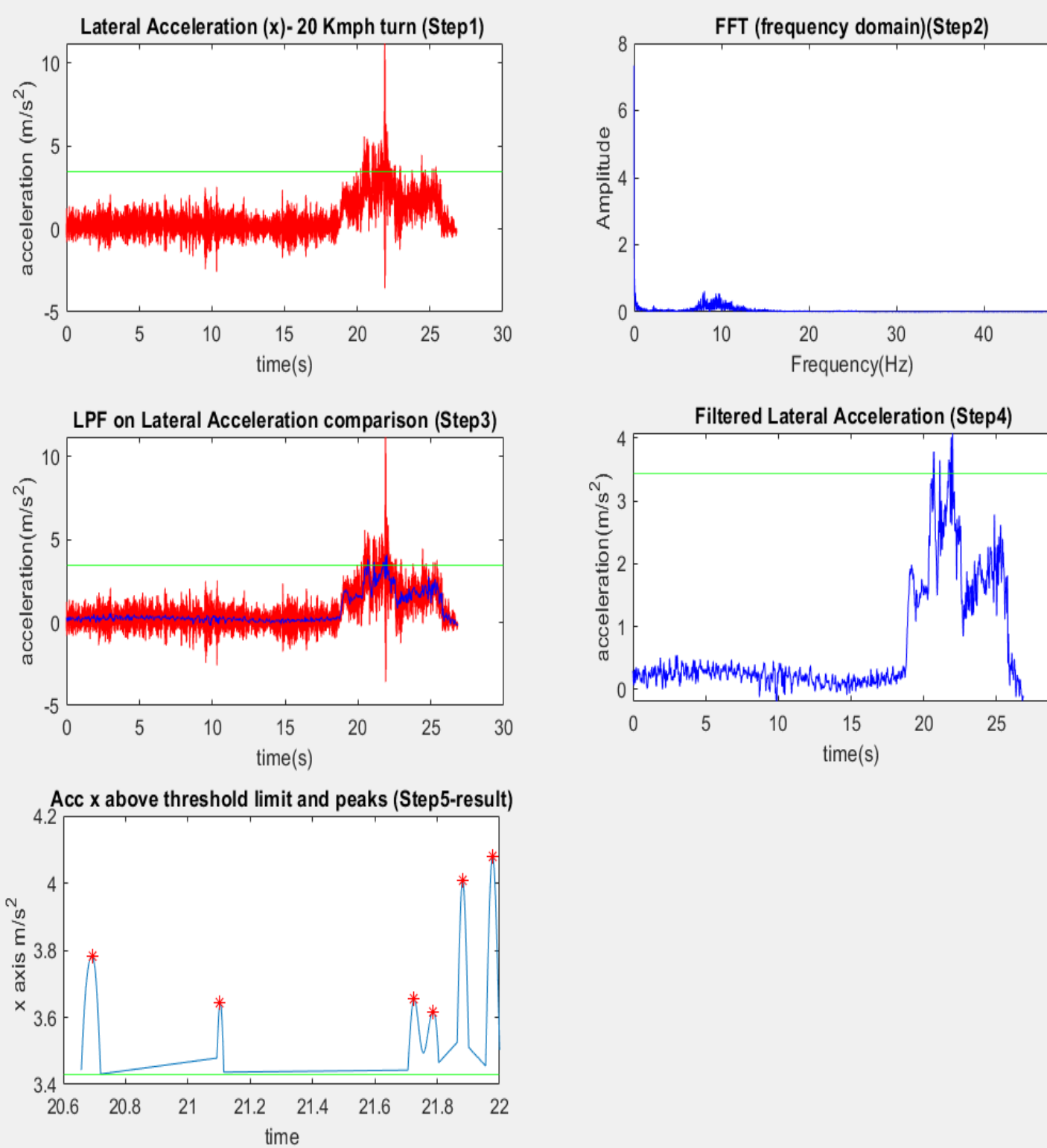

Fig.11 At $20 \mathrm{Kmph}$ turn complete output graph

Figure 11 depicts a scenario where the vehicle takes a turn at $20 \mathrm{Kmph}$ and the signal acquired from this event is processed by the algorithm which is shown graphically step by step. The red signal is unfiltered raw data and the blue signal is the filtered signal. The green line represents the threshold value $(0.35 \mathrm{~g})$ for acceleration above which is considered to be rash driving. This is considered to be a rash driving event as the signal crosses the threshold limit. 

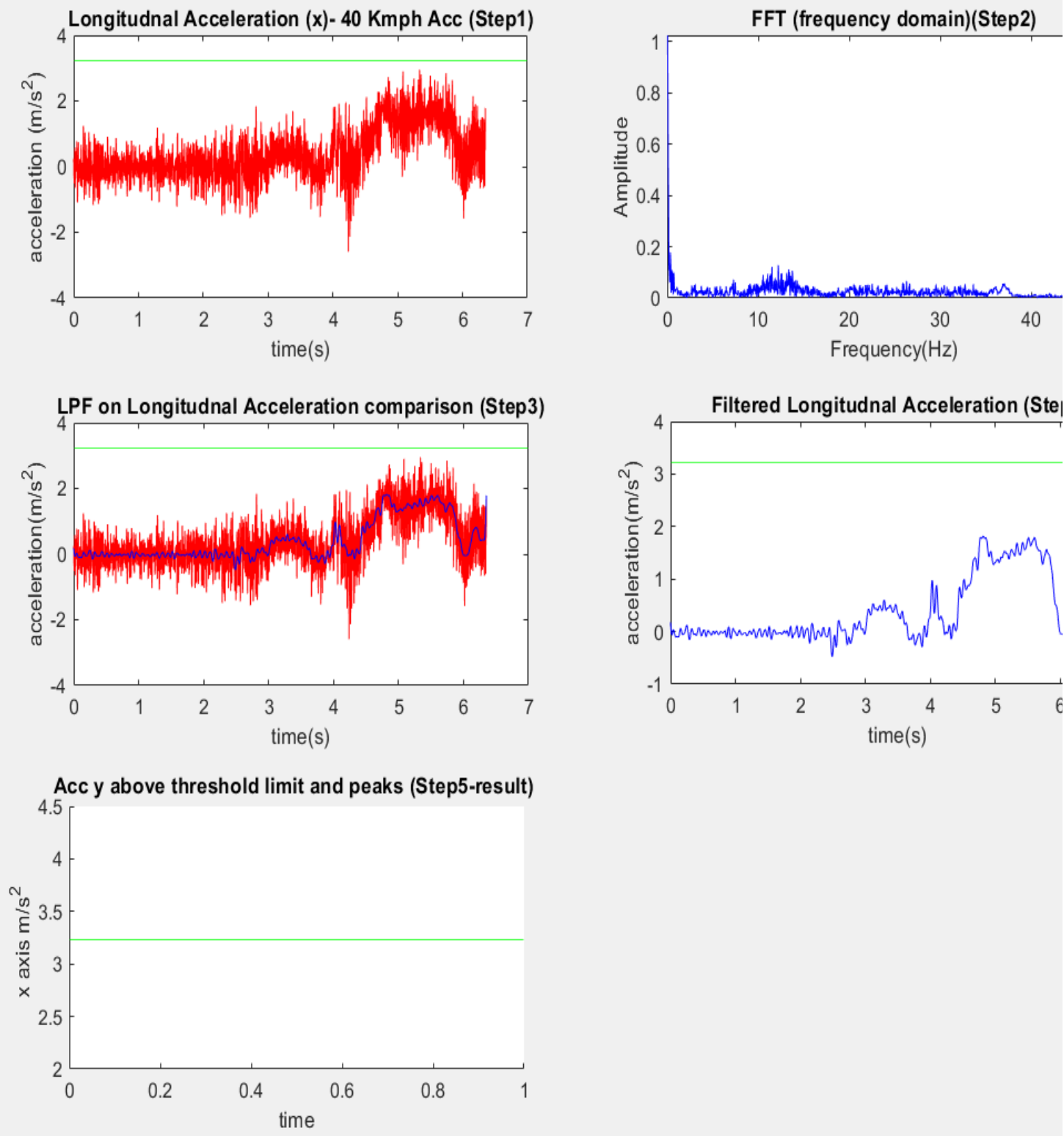

Fig.12 At $40 \mathrm{Kmph}$ Acceleration complete output graph

Figure 12 depicts a scenario where the vehicle accelerates from $0-40 \mathrm{Kmph}$ and the signal acquired from this event is processed by the algorithm which is shown graphically step by step. The red signal is unfiltered raw data and the blue signal is the filtered signal. The green line represents the threshold value $(0.33 \mathrm{~g})$ for acceleration above that is considered to be rash driving. 

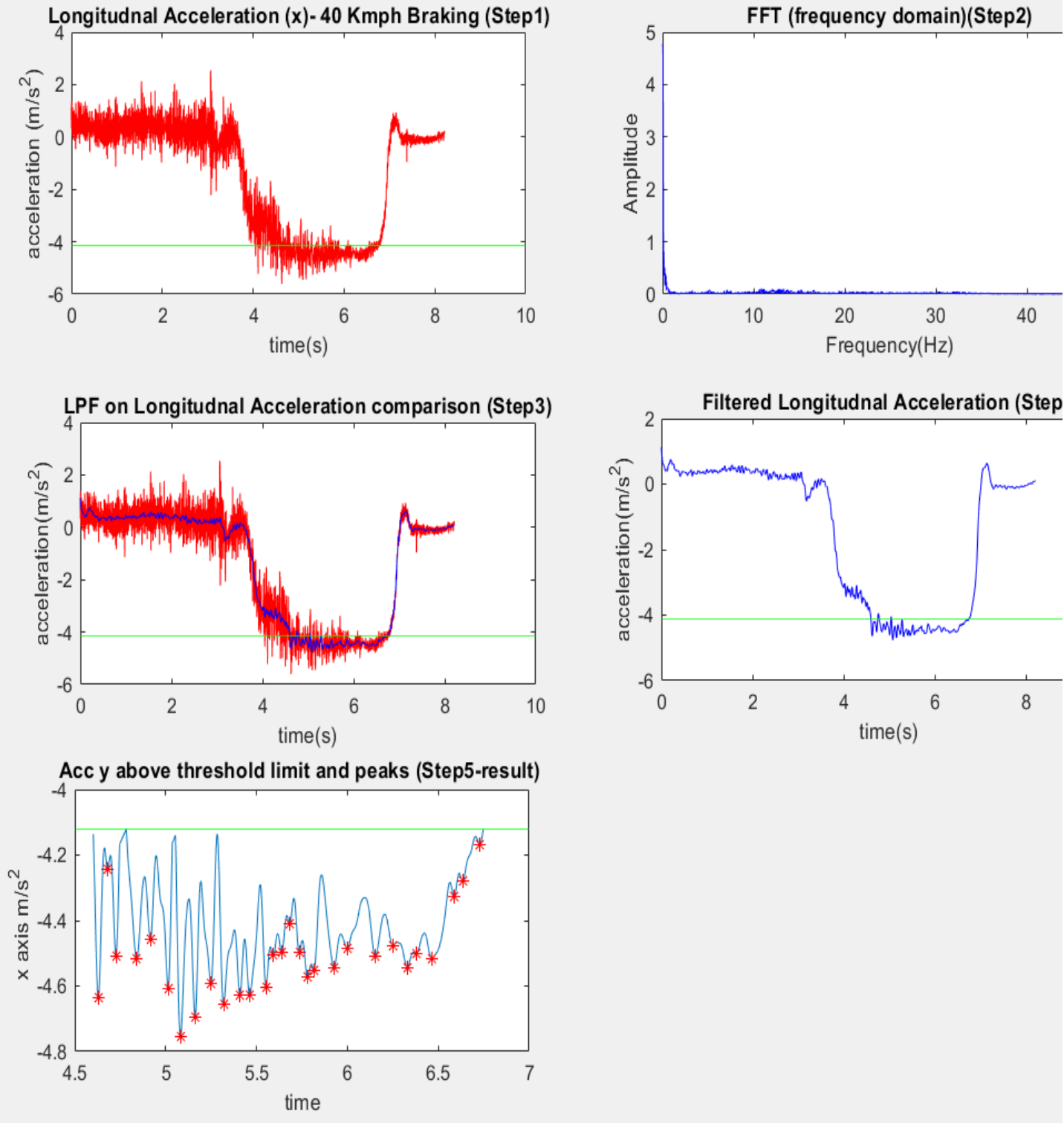

Fig.13 At $40 \mathrm{Kmph}$ braking complete output graph

Figure 13 depicts a scenario where the vehicle brakes from 40-0 Kmph and the signal acquired from this event is processed by the algorithm which is shown graphically step by step. The red signal is unfiltered raw data and the blue signal is the filtered signal. The green line represents the threshold value $(0.42 \mathrm{~g})$ for acceleration above which is considered to be rash driving. This is considered to be a rash driving event as the signal crosses the threshold limit. 

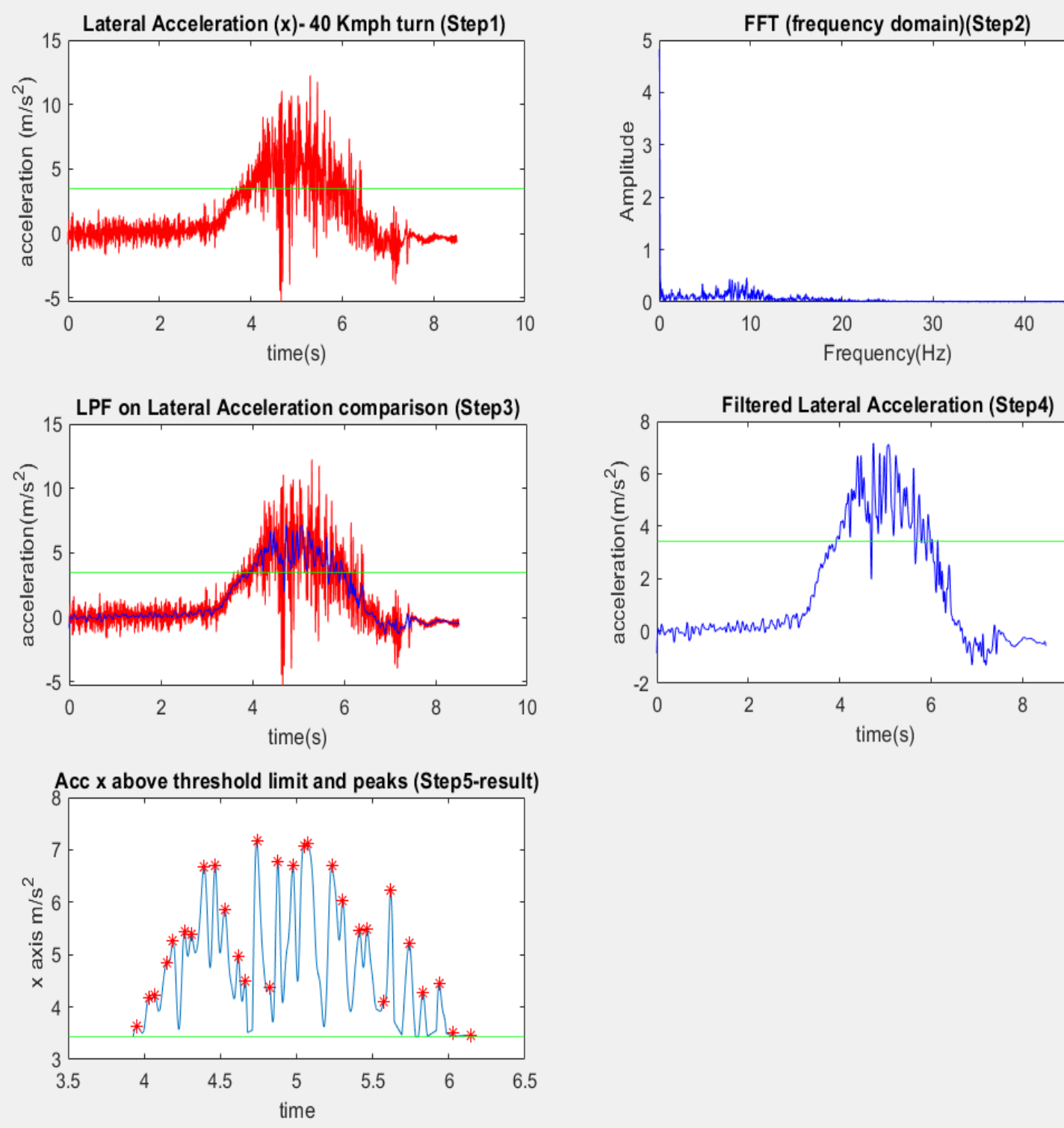

Fig.14 At 40 Kmph turn complete output graph

Figure 14 depicts a scenario the vehicle takes a turn at $40 \mathrm{Kmph}$ and the signal acquired from this event is processed by the algorithm which is shown graphically step by step. The red signal is unfiltered raw data and the blue signal is the filtered signal. The green line represents the threshold value $(0.35 \mathrm{~g})$ for acceleration above which is considered to be rash driving. This is considered to be a rash driving event as the signal crosses the threshold limit.

\section{6}

\section{. Conclusion}

With the increase in population and the number of vehicles on the road it has become more crucial to ensure safety of the passengers, Driver and pedestrians. Using the powerful smartphones to record the driving data and feeding it into the developed algorithm to identify rash driving and crashes and immediately and warning the driver and concerned authorities for swift action and ensuring a safe road environment.

$>$ The concept of using smartphones to record the driving data and using a custom made algorithm to analyse it and detect the driving pattern is crucial for countries like India where the 
development rate is so high its sometimes difficult to monitor and maintain order everytime. The population density and the ever growing number of vehicles on road is a factor which can put the very traffic infrastructure and road safety at risk if tradition methods that ensure its integrity can't keep up. This solution of using something that is very cost effective and easy to implement is a great advantage.

The algorithm is capable to detect driving pattern and pin point dangerous driving and accidents with good accuracy and is able to support multiple sources if the need is there like an independent accelerometer sensor with raspberry pi or arduino in the entire system acts as a blackbox. The app also complements the entire platform by providing initial warnings which help in preventing incidents to a certain degree by alarming the driver and at the same time records the data from the inbuilt accelerometer sensor.

In this research, the successful development of an algorithm is able to detect driving pattern and with a good amount of accuracy with primary focus towards compact SUV as it's the most popular segment at present and an primary app which is able to record data and give the driver initial warning were developed. The core of the algorithm is the same for all vehicle types and to accommodate for other vehicle types just a minor adjustment to the threshold limit is required for that specific type of vehicle.

\section{References}

[1] Kalra, Nidhi, and Divya Bansal. "Analyzing driver behavior using smartphone sensors: a survey." Int. J. Electron. Electr. Eng 7, no. 7 (2014): 697-702.

[2] Hong, Jin-Hyuk, Ben Margines, and Anind K. Dey. "A smartphone-based sensing platform to model aggressive driving behaviors." In Proceedings of the SIGCHI Conference on Human Factors in Computing Systems, pp. 4047-4056. 2014.

[3] Gharibeh, Hamed Farhadi, Ahmad Sadeghi Yazdankhah, and Mohammad Reza Azizian. "Energy management of fuel cell electric vehicles based on working condition identification of energy storage systems, vehicle driving performance, and dynamic power factor." Journal of Energy Storage 31 (2020): 101760.

[4] Sarker, Ankur, Chenxi Qiu, Haiying Shen, Hua Uehara, and Kevin Zheng. "Brake Data-Based Location Tracking in Usage-Based Automotive Insurance Programs." In 2020 19th ACM/IEEE International Conference on Information Processing in Sensor Networks (IPSN), pp. 229-240. IEEE, 2020.

[5] Al-Din, Munaf Salim Najim, and Atef Saleh Al-Mashakbeh. "Development of a highway driving events identification and classification using smartphone." International Journal of Nanoparticles 12, no. 1-2 (2020): 152-173.

[6] Yassine Graduate Engineer, Zein. "FUZZY LOGIC VELOCITY OPTIMIZATION OF AUTONOMOUS VEHICLES BASED ON ROAD BUMP GEOMETRY." BAU Journal-Science and Technology 1, no. 2 (2020): 2.

[7] Urmson, Christopher Paul, Dmitri A. Dolgov, and Philip Nemec. "Driving pattern recognition and safety control." U.S. Patent 8,965,621, issued February 24, 2015.

[8] Zheng, Yang, and John HL Hansen. "Unsupervised driving performance assessment using free-positioned smartphones in vehicles." In 2016 IEEE 19th International Conference on Intelligent Transportation Systems (ITSC), pp. 1598-1603. IEEE, 2016.

[9] Johnson, Derick A., and Mohan M. Trivedi. "Driving style recognition using a smartphone as a sensor platform." In 2011 14th International IEEE Conference on Intelligent Transportation Systems (ITSC), pp. 1609-1615. IEEE, 2011.

[10] Sunkara, S.C., Balaji, R. and Babu, M., 2020. A critical investigation on ultrasound cyber-attack and using fourier transform for defence application against inaudible-attacks. Materials Today: Proceedings.

[11] Alekhya, G.N., Abhishikt, C.B.N.S. and Ramachandran, B., 2020. A recent investigation on design aspects of vehicle crash analysis in India. Materials Today: Proceedings, 33, pp.4479-4485. 
[12] Kodam, S., Bharathgoud, N. and Ramachandran, B., 2020. A review on smart wearable devices for soldier safety during battlefield using WSN technology. Materials Today: Proceedings, 33, pp.4578-4585.

[13] Zhang, Z., Zhang, H., Xu, S. and Lv, W., 2021. Pavement roughness evaluation method based on the theoretical relationship between acceleration measured by smartphone and IRI. International Journal of Pavement Engineering, pp.1-17. 